\title{
Hallazgos ecográficos para la determinación de nódulos tiroideos malignos
}

\author{
Ultrasound findings for the determination of \\ malignant thyroid nodules
}

\author{
Johana A Guarnizo $\mathrm{V}^{1}$ (D)
}

Forma de citar: Guarnizo J, Ortiz S, Núñez J, Trujillo I. Hallazgos ecográficos para la determinación de nódulos tiroideos malignos. Salud UIS. 2021; e21026. doi: https://doi.org/10.18273/saluduis.53.e:21026 @) (i)

\section{Resumen}

Introducción: los nódulos tiroideos son un crecimiento localizado en el tejido tiroideo, aproximadamente el $8 \%$ son malignos, y el ultrasonido es el método ideal para detectarlos. Objetivo: determinar cuáles son los hallazgos ecográficos que pueden sugerir una mayor probabilidad de malignidad del nódulo tiroideo. Metodología: estudio descriptivo de tipo corte transversal en pacientes con nódulo tiroideo en una institución prestadora de salud de Neiva. Resultados: se evaluaron 63 pacientes con un promedio de edad de 52 años, $95 \%$ fueron femeninos y el $5 \%$ masculinos. El $71 \%$ presentaron nódulos tiroideos benignos, y el 10\% nódulos malignos. En el $100 \%$ de los nódulos tiroideos malignos se encontró vascularidad aumentada, microcalcificaciones, adenopatías asociadas, bordes irregulares y componente sólido, y los hallazgos con respecto a hipoecogenicidad y diámetro anteroposterior mayor al transverso (más alto que ancho), se encontró en el $83 \%$, siendo estadísticamente significativos. La sensibilidad de los hallazgos ecográficos supera el $80 \%$, excepto la variable "tamaño del nódulo mayor a 1 centímetro". Conclusiones: Los hallazgos ecográficos como adenopatías y el diámetro anteroposterior mayor al transverso son indicadores potenciales de nódulos tiroideos malignos, el tamaño mayor a un centímetro no siempre es predictor de malignidad, sin embargo debido al tamaño de la muestra en nuestro estudio no es posible generalizarlo como un factor determinante para la realización de biopsia, por ende recomendamos el seguimiento de los nódulos teniendo en cuenta los criterios ecográficos de malignidad y la clasificación TIRADS para tomar decisiones con respecto a las biopsias tiroideas.

Palabras clave: Nódulo Tiroideo; Cáncer de Tiroides; Ecografía de Tiroides; Citología; Biopsia.

\begin{abstract}
Introduction: Thyroid nodules are a localized growth in the thyroid tissue, approximately $8 \%$ are malignant, and an ultrasound is the ideal method to detect them. Objective: Determine which are the ultrasound findings that may suggest an increased probability of thyroid nodule malignancy. Methodology: Descriptive cross-sectional study amongst patients with thyroid nodule in a healthcare institution in Neiva. Results: A total of 63 patients

1. Universidad Surcolombiana. Neiva, Colombia.

2. Universidad Nacional de la Plata. Argentina.

3. Fundación Universitaria Sanitas. Bogotá, Colombia.

4. Universidad Surcolombiana. Neiva, Colombia.

Correspondencia: Johana Alejandra Guarnizo. Dirección: calle 48 n. 20-108 prado norte Neiva. Teléfono: +57 3204085269. Correo electrónico: alejitaguarnizo@hotmail.com
\end{abstract}


were evaluated with an average age of 52 years, $95 \%$ were female and $5 \%$ male. Seventy-one percent had benign thyroid nodules, and 10\% malignant nodules. In $100 \%$ of malignant thyroid nodules, increased vascularity, microcalcifications, associated adenopathies, irregular borders and solid component were found, and the findings regarding hypoechogenicity and anteroposterior diameter greater than the transverse (higher than wide), were found in $83 \%$, being statistically significant. The sensitivity of ultrasound findings exceeds $80 \%$, except for the variable nodule size greater than 1 centimetre. Conclusions: Ultrasound findings such as adenopathies and anteroposterior diameters greater than the transverse one are potential indicators of malignant thyroid nodules, the size greater than one centimetre is not always a predictor of malignancy; however, due to the size of the sample in our study it is not possible to generalize it as a determining factor for biopsy. Therefore, we recommend monitoring the nodules considering the ultrasound criteria for malignancy and the TIRADS classification to make decisions regarding thyroid biopsies.

Keywords: Thyroid Nodule; Thyroid Cancer; Thyroid Ultrasound; Cytology; Biopsy.

\section{Introducción}

Los nódulos tiroideos son un crecimiento localizado en el tejido tiroideo (nódulo único) acompañado o no del crecimiento del resto de la tiroides. En la ecografía son lesiones intratiroideas radiológicamente distintas al parénquima que las rodea, su prevalencia depende del método que se utilice ${ }^{1}$, por medio de palpación está entre 4 y el $7 \%$ y por ecografía entre el 20 al $76 \%$, el cáncer de tiroides cada vez tiene una incidencia más elevada, aproximadamente el 4 al $8 \%$ de los nódulos son cancerígenos ${ }^{2}$. La ecografía es el instrumento de elección para estudio de los nódulos tiroideos, no obstante, no proporciona suficiente información para la confirmación de malignidad del nódulo; debido a que es un examen operador dependiente, la descripción por radiólogos no es la misma y la clasificación Tirads ${ }^{3}$ no siempre es reportada. Por lo tanto, la Biopsia por Aspiración Con Aguja Fina (BACAF) se ha considerado como necesaria para establecer la malignidad del Nódulo ${ }^{4,5}$, a pesar de su eficacia, no es fácil ni oportuno obtener un procedimiento mínimamente invasivo como este, en la menor brevedad posible evitando morbilidad y mortalidad. Por consiguiente, el siguiente trabajo tiene como objetivo establecer la sensibilidad y especificidad de los hallazgos ecográficos para la determinación de la malignidad de los nódulos tiroideos en una institución prestadora de salud ubicada en el municipio de Neiva con el fin de mejorar el enfoque diagnóstico y de tratamiento en pacientes con nódulo tiroideo en nuestra región.

\section{Metodología}

En el presente estudio se revisaron historias clínicas de pacientes mayores de edad sin diagnóstico previo de cáncer de tiroides, que consultaron a la entidad prestadora de servicio de salud de Neiva, durante el periodo 2014-
2019 a quienes se les realizó ecografía por sospecha de nódulo tiroideo, y que posteriormente se confirmó mediante BACAF. Para determinar la efectividad de los hallazgos ecográficos, se calculó la sensibilidad, especificidad, Valor Predictivo Positivo (VPP) y Valor Predictivo Negativo (VPN) de las características ecográficas que sugieren malignidad (vascularidad central aumentada, microcalcificación, adenopatías, hipoecogenicidad, componente sólido(nódulo quístico con componente sólido), bordes irregulares, diámetro anteroposterior mayor que el transverso y tamaño del nódulo mayor a $1 \mathrm{~cm}$ ), establecidas por la Asociación Americana de Tiroides (ATA) ${ }^{6}$. El resultado de la biopsia es categorizado con el sistema de Bethesda, que tiene 6 categorías: I no diagnostica, II benigna, III atipia/lesión folicular de significado indeterminado, IV neoplasia folicular/sospecha de neoplasia folicular, V sospecha de malignidad, VI maligna ${ }^{7}$.

La caracterización de los pacientes y de los hallazgos ecográficos se realizó con el total de los pacientes, que cumplieron los criterios de inclusión siendo estos: pacientes con diagnóstico de nódulo tiroideo (CIE -10 E041) encontrados en la base de datos de la IPS y pacientes mayores de 18 años y criterios de exclusión: pacientes con diagnóstico previo de cáncer de tiroides, pacientes que no contaran con la información completa en la historia clínica, como lo son el reporte de la ecografía y el reporte de la biopsia. Para la determinación de la sensibilidad, especificidad, VPP y VPN, solo se realizó en los pacientes con nódulos benignos (Bethesda II) y malignos (Bethesda VI). Los datos fueron registrados y procesados en el software STATA versión 13.

Este trabajo fue previamente evaluado y aprobado por el comité de ética de la universidad Surcolombiana mediante sesión ordinaria del 13 de febrero del 2020 y según consta en acta No.001. 


\section{Resultados}

Se revisaron 85 historias clínicas, de las cuales se excluyeron 22 , por información incompleta: falta de reporte ecográfico o de biopsia, o que no cumplían con criterios de inclusión, por lo que finalmente se evaluaron 63 pacientes, con un promedio de edad de 52 años, y un índice de masa corporal promedio de 26 $\left(\mathrm{kg} / \mathrm{m}^{2}\right)$, con respecto al sexo el $95 \%$ fueron femeninos y el $5 \%$ masculinos. Según el sitio de procedencia el $58 \%$ de ellos pertenecen a la ciudad donde se ubica la institución prestadora de salud. Se observó que el $71 \%$ de los pacientes con diagnóstico mediante BACAF, corresponden a nódulos tiroideos benignos, el 10\% a nódulos malignos y el $19 \%$ corresponden a los grupos I, III, IV y V respectivamente, según el sistema de clasificación Bethesda como se evidencia en la tabla 1.

Tabla 1. Características sociodemográficas y clasificación Bethesda.

\begin{tabular}{lcc}
\hline \multicolumn{1}{c}{ Variable } & Nivel de la variable & Valor $\mathbf{n}(\%)$ \\
$\mathrm{N}$ & & 63 \\
Edad, mean (SD) & & $52,76(13,91)$ \\
IMC $^{\mathrm{b}}$, mean (SD) & $\mathrm{F}^{\mathrm{c}}$ & $26,06(4,97)$ \\
Sexo & $\mathrm{M}^{\mathrm{d}}$ & $60(95)$ \\
Clasificación & $\begin{array}{c}\text { Atipia de significado } \\
\text { indeterminado (III) }\end{array}$ & $1(2)$ \\
Bethesda & $\begin{array}{c}\text { Benigno (II) } \\
\text { Maligno (VI) }\end{array}$ & $45(71)$ \\
& $\begin{array}{c}\text { Neoplasia folicular } \\
\text { (IV) }\end{array}$ & $3(10)$ \\
& $\begin{array}{c}\text { No diagnóstico } \\
\text { insatisfactorio (I) } \\
\text { Sospechoso de } \\
\text { malignidad (V) }\end{array}$ & $6(10)$ \\
& & $2(3)$ \\
\hline
\end{tabular}

a SD: desviación estándar.

${ }^{\mathrm{b}}$ IMC: índice de masa corporal.

${ }^{c} \mathrm{~F}$ : femenino.

${ }^{\mathrm{d}}$ M: masculino.

Fuente propia.

Al evaluar los hallazgos durante la ecografía, en la tabla 2 , se observa que el $89 \%$ de los pacientes presentaban tamaño del nódulo tiroideo mayor a $1 \mathrm{~cm}$. Respecto a los parámetros ecográficos asociados a malignidad el más frecuente fue la hipoecogenicidad en el $40 \%$, seguido por componente sólido en el $38 \%$, el $35 \%$ presentó bordes irregulares, vascularidad aumentada en el $32 \%$, el $24 \%$ presentaba microcalcificaciones, adenopatías en el $22 \%$, y en menor frecuencia el diámetro anteroposterior (AP) mayor transverso que correspondió al $14 \%$.
Tabla 2. Hallazgos ecográficos de los pacientes con nódulo tiroideo.

\begin{tabular}{|c|c|c|}
\hline Variable & Nivel de la variable & $\mathbf{N}(\%)$ \\
\hline \multirow{3}{*}{ Vascularidad } & Aumentada & $20(32)$ \\
\hline & Ausente & $28(44)$ \\
\hline & Escasa & $15(24)$ \\
\hline \multirow{2}{*}{ Microcalcificaciones } & No & $48(76)$ \\
\hline & Sí & $15(24)$ \\
\hline \multirow{2}{*}{ Adenopatías asociadas } & No & $49(78)$ \\
\hline & Sí & $14(22)$ \\
\hline \multirow{2}{*}{ Hipoecogenicidad } & No & $38(60)$ \\
\hline & Sí & $25(40)$ \\
\hline \multirow{2}{*}{$\begin{array}{l}\text { Bordes irregulares o } \\
\text { microlobulados }\end{array}$} & No & $41(65)$ \\
\hline & Sí & $22(35)$ \\
\hline \multirow{2}{*}{ Componente sólido } & No & $39(62)$ \\
\hline & Sí & $24(38)$ \\
\hline \multirow{2}{*}{ Tamaño nódulo } & Mayor & $56(89)$ \\
\hline & Menor & 7 (11) \\
\hline \multirow{2}{*}{$\begin{array}{l}\text { Diámetro ap }>\text { al } \\
\text { transverso }\end{array}$} & No & $54(86)$ \\
\hline & Sí & $9(14)$ \\
\hline
\end{tabular}

Fuente: Propia.

Se realizó la comparación de las características sociodemográficas y los hallazgos ecográficos entre los pacientes que presentaron una clasificación Bethesda II (benigno) y VI (maligno), evidentes en la tabla 3. En los pacientes con nódulos tiroideos benignos se encontró que el $93 \%$ eran de sexo femenino y el $7 \%$ masculino, esto evidencia el predominio de esta patología en el género femenino tanto en nódulos benignos como en malignos. Se encontró una mediana para la edad de 55,1 y 49,3 respectivamente en nódulos benignos y malignos. Con respecto al IMC se encontró una mediana de $25,54\left(\mathrm{~kg} / \mathrm{m}^{2}\right)$ en nódulos benignos y de $27,57\left(\mathrm{~kg} / \mathrm{m}^{2}\right)$ en nódulos malignos. El tamaño del nódulo mayor a $1 \mathrm{~cm}$ fue del $93 \%$ y $67 \%$, respectivamente. En el $100 \%$ de los nódulos tiroideos malignos se encontró vascularidad aumentada, microcalcificaciones, adenopatías asociadas, bordes irregulares o microlobulados y componente sólido. Los hallazgos con respecto a hipoecogenicidad y diámetro AP mayor al transverso, se encontraron en el $83 \%$, siendo estadísticamente significativos. 
Tabla 3. Hallazgos Ecográficos en nódulos benignos (II) y malignos (VI).

\begin{tabular}{|c|c|c|c|c|c|}
\hline Variable & $\begin{array}{c}\text { Nivel de la } \\
\text { variable }\end{array}$ & Benigno (\%) & Malignos (\%) & $\begin{array}{c}\text { OR } \\
\text { IC 95\% }\end{array}$ & p-value \\
\hline $\mathrm{N}$ & & $45(71)$ & $6(10)$ & & \\
\hline edad, Mean $(\mathrm{SD})^{\mathrm{b}}$ & & $55,11(12,90)$ & $49,33(9,16)$ & - & 0,30 \\
\hline $\mathrm{IMC}^{\mathrm{c}}$, Mean (SD) & & $25,54(4,37)$ & $27,57(6,9)$ & - & 0,32 \\
\hline \multirow{2}{*}{ Sexo } & Femenino & $42(93)$ & $6(100)$ & \multirow{2}{*}{ - } & \multirow{2}{*}{1,00} \\
\hline & Masculino & $3(7)$ & $0(0)$ & & \\
\hline \multirow{3}{*}{ Vascularidad } & Aumentada & $11(24)$ & $6(100)$ & \multirow{3}{*}{ - } & \multirow{3}{*}{0,002} \\
\hline & Ausente & $23(51)$ & $0(0)$ & & \\
\hline & Escasa & $11(24)$ & $0(0)$ & & \\
\hline \multirow{2}{*}{ Microcalcificaciones } & No & $41(91)$ & $0(0)$ & \multirow[b]{2}{*}{ - } & \multirow{2}{*}{$<0,001$} \\
\hline & $\mathrm{Si}$ & $4(9)$ & $6(100)$ & & \\
\hline \multirow{2}{*}{ Adenopatías asociadas } & No & $44(98)$ & $0(0)$ & \multirow{2}{*}{ - } & \multirow{2}{*}{$<0,001$} \\
\hline & $\mathrm{Si}$ & $1(2)$ & $6(100)$ & & \\
\hline \multirow{2}{*}{ Hipoecogenicidad } & No & $31(69)$ & $1(17)$ & \multirow{2}{*}{$\begin{array}{c}11,07 \\
(1,18-103,77)\end{array}$} & \multirow{2}{*}{0,022} \\
\hline & $\mathrm{Si}$ & $14(31)$ & $5(83)$ & & \\
\hline \multirow{2}{*}{$\begin{array}{l}\text { Bordes irregulares o } \\
\text { microlobulados }\end{array}$} & No & $34(76)$ & $0(0)$ & \multirow{2}{*}{-} & \multirow{2}{*}{$<0,001$} \\
\hline & $\mathrm{Si}$ & $11(24)$ & $6(100)$ & & \\
\hline \multirow{2}{*}{ Componente sólido } & No & $35(78)$ & $0(0)$ & \multirow{2}{*}{-} & \multirow{2}{*}{$<0,001$} \\
\hline & $\mathrm{Si}$ & $10(22)$ & $6(100)$ & & \\
\hline \multirow{2}{*}{ Tamaño del nódulo. } & Mayor a $1 \mathrm{~cm}$. & $42(93)$ & $4(67)$ & 7 & \multirow{2}{*}{0,099} \\
\hline & Menor a $1 \mathrm{~cm}$. & $3(7)$ & $2(33)$ & $(0,89-55,04)$ & \\
\hline \multirow{2}{*}{ Diámetro ap $^{\mathrm{d}}>$ al transverso } & No & $44(98)$ & $1(17)$ & \multirow{2}{*}{$\begin{array}{c}220,00 \\
(11,84-4087,55)\end{array}$} & \multirow{2}{*}{$<0,001$} \\
\hline & $\mathrm{Si}$ & $1(2)$ & $5(83)$ & & \\
\hline
\end{tabular}

${ }^{a}$ Comparación con $\mathrm{t}$ student y las $\mathrm{p}$ de las variables categóricas con test exacto de Fisher.

b SD: desviación estándar.

c IMC: índice de masa corporal.

$\mathrm{d}$ ap: anteroposterior.

Fuente propia.

Se evidenció que la sensibilidad de los hallazgos ecográficos supera el $80 \%$, con intervalos de confianza $95 \%$ entre 91 y 100, con especificidad superior al $68 \%$, con intervalos de confianza $95 \%$ entre 61 y 100 , excepto la variable "tamaño del nódulo mayor a $1 \mathrm{~cm}$ ", que tiene una sensibilidad del $66,7 \%$ y una especificidad muy inferior en comparación con los otros hallazgos. Se encontró que la mayor parte de los valores predictivos positivos (VPP) fueron bajos, a diferencia de las microcalcificaciones, adenopatías y diámetro AP mayor al transverso que presentaron VPP altos, los demás hallazgos presentaron VPN mayor a $96 \%$ con intervalos de confianza $95 \%$ entre 89 y 100 , como se logran apreciar en la tabla 4. Dentro de los hallazgos se puede sugerir que las adenopatías y el diámetro AP mayor al transverso son las variables de mejor rendimiento diagnóstico para la confirmación de nódulos malignos mediante BACAF debido a su VPP superior y especificidad. 
Tabla 4. Sensibilidad y especificidad de hallazgos ecográficos según el reporte de BACAF en nódulos malignos.

\begin{tabular}{|c|c|c|c|c|c|c|c|c|}
\hline \multirow{2}{*}{ Hallazgo ecográfico } & \multicolumn{2}{|c|}{ Sensibilidad } & \multicolumn{2}{|c|}{ Especificidad } & \multicolumn{2}{|r|}{ VPP } & \multicolumn{2}{|r|}{$\mathbf{V P N} \mathbf{N}^{\mathrm{b}}$} \\
\hline & $(\%)$ & IC $95 \%$ & $(\%)$ & IC $95 \%$ & $(\%)$ & IC $95 \%$ & $(\%)$ & IC $95 \%$ \\
\hline Microcalcificaciones & 100 & $91,67-100,00$ & 91,1 & $81,69-100,00$ & 60 & $24,64-95,36$ & 100 & $98,78-100,00$ \\
\hline Adenopatías asociadas & 100 & $91,67-100,00$ & 97,8 & $92,36-100,00$ & 85,7 & $52,65-100,00$ & 100 & $98,86-100,00$ \\
\hline Hipoecogenicidad & 83,3 & $45,18-100,00$ & 68,9 & $54,25-83,53$ & 26,3 & $3,88-48,75$ & 96,9 & $89,28-100,00$ \\
\hline $\begin{array}{l}\text { Bordes irregulares o } \\
\text { microlobulados }\end{array}$ & 100 & $91,67-100,00$ & 75,6 & $61,89-89,22$ & 35,3 & $9,64-60,95$ & 100 & $98,53-100,00$ \\
\hline Componente sólido & 100 & $91,67-100,00$ & 77,8 & $64,52-91,04$ & 37,5 & $10,65-64,35$ & 100 & $98,57-100,00$ \\
\hline Diámetro $\mathrm{ap}^{\mathrm{c}}>$ al transverso & 83,3 & $45,18-100,00$ & 97,8 & $92,36-100,00$ & 83,3 & $45,18-100,00$ & 97,8 & $92,36-100,00$ \\
\hline Vascularidad aumentada & 100 & $91,67-100,00$ & 75,6 & $61,89-89,22$ & 35,3 & $9,64-60,95$ & 100 & $98,53-100,00$ \\
\hline Tamaño $>1 \mathrm{~cm}$ & 66,7 & $20,61-100,0$ & 6,67 & $0,00-15,07$ & 8,7 & $0,00-17,93$ & 60 & $7,06-100,00$ \\
\hline
\end{tabular}

${ }^{a}$ VPP: valor predictivo positivo.

${ }^{\mathrm{b}} \mathrm{VPN}$ : valor predictivo negativo.

${ }^{c}$ ap: anteroposterior.

Fuente propia.

\section{Discusión}

Se evaluó los hallazgos ecográficos para la determinación de la malignidad de los nódulos tiroideos, y se encontró una sensibilidad del $83 \%$ al $100 \%$ y especificidad del $68 \%$ al $97 \%$, muy similar a la de otros estudios ${ }^{8}$. Sin embargo, los VPP son significativamente más bajos que los reportados en la literatura científica, esto puede explicarse debido a que la prevalencia de nódulos tiroideos malignos en la población estudiada fue muy baja. Según este estudio el tamaño de los nódulos no fue útil para distinguir entre nódulos benignos y malignos, fue el hallazgo de menor sensibilidad y especificidad ya que el tamaño en la mayoría, tanto de los benignos como de los malignos, fue mayor a $1 \mathrm{~cm}$, de hecho, se ha encontrado controversia sobre si realmente este sea un hallazgo predictor de malignidad ya que muchos pacientes con cáncer de tiroides, inician con un nódulo incidental menor a 1 centímetro, ${ }^{9,10}$, algunos investigadores han sugerido una relación positiva entre el tamaño y el riesgo de malignidad ${ }^{11,12}$ pero otros han informado que el tamaño del nódulo no es predictivo de ésta ${ }^{13,14}$. Debido al tamaño de la muestra en nuestro estudio no es posible generalizar el tamaño como un factor determinante para la realización de biopsia, de hecho se sugiere que esta se realice solo en nódulos que tengan riesgo alto de malignidad, según sus patrones ecográficos.

La hipoecogenicidad del nódulo descrita por múltiples autores como uno de los factores de riesgo más importante de asociación con cáncer $^{15}$ fue estadísticamente significativa, sin embargo, presenta un valor predictivo positivo bajo. Al igual que lo encontrado por otros grupos, la vascularidad aumentada, fue mucho mayor en el nódulo maligno $\mathrm{y}$ muy ocasional en los benignos ${ }^{16}$ en el presente trabajo $100 \%$ de los nódulos malignos la presentaban. Similar a la mayoría de los estudios los bordes irregulares o microlobulados, componente sólido y microcalcificaciones sugieren neoplasia maligna, en esta investigación fueron significativos, no obstante, Chala, et al. ${ }^{17}$ encontraron que las microcalcificaciones fueron estadísticamente significativas y tuvieron un nivel moderado de concordancia, solo al asociarlas con el componente sólido y la vascularidad aumentada. En otros estudios se evidenció que la detección de 
calcificaciones en la ecografía aumenta el índice clínico de sospecha de carcinoma de tiroides y tiene una especificidad muy alta para predecirlo, particularmente para aquellos pacientes menores de 45 años o con ganglios linfáticos regionales calcificados ${ }^{18}$, esta relación es importante debido a que en este trabajo las microcalcificaciones y las adenopatías mostraban una especificidad de $91,1 \%$ y $97,8 \%$ respectivamente, lo que permiten un mejor rendimiento diagnóstico.

En este estudio se presentaron dos hallazgos como los mejores predictores de malignidad, el primero: las adenopatías asociadas al igual que el estudio realizado por Oliveira, et al. ${ }^{19}$ Según autores las metástasis ganglionares regionales del cuello están presentes hasta en el $50-70 \%$ de los casos de malignidad, incluidas aquellas metástasis microscópicas halladas en la histología, por lo tanto, puede considerarse como una situación frecuente. Según Mac Dermott, et al. el compromiso ganglionar no es considerado un factor de riesgo importante de mortalidad, excepto en pacientes mayores de 45 años, en quienes su presencia sí modifica la estadificación de riesgo de mortalidad, tratamiento y seguimiento $^{20}$. Y el segundo es el diámetro AP mayor al transverso, con alta sensibilidad, especificidad del $97,8 \%$ y altos valores predictivos, muy similar a como se describe en otros estudios donde esta asociación alcanza el $97 \%^{21,22}$. Ren, et al. informaron que un diámetro AP mayor al transverso fue la característica ecográfica más precisa para predecir carcinoma papilar de tiroides en nódulos pequeños y si se asocia a otra característica ecográfica se logra mayor precisión diagnóstica ${ }^{23}$.

En cuanto a las limitaciones del estudio fueron algunos datos incompletos en las historias clínicas, por lo cual se tuvieron que excluir algunos pacientes y así disminuir el número de la muestra, además es probable un sesgo de información secundario a un probable mal diligenciamiento en la historia clínica o por falta de datos confiables y el sesgo de centro de referencia por ser la única institución de la que se obtuvo la información. Sin embargo, a nivel regional y local, es el primer estudio realizado, lo que aporta una información valiosa sobre nuestra población. Este estudio servirá como un marco referencial para generar nuevas investigaciones y estudios multicéntricos, sin embargo, debido a que el tamaño de la muestra fue pequeño, se sugiere realizar un estudio de cohorte o controlado aleatorizado para mejorar la hipótesis y realizar un seguimiento de los pacientes a través del tiempo en cuanto a su evolución y las ayudas diagnósticas.

\section{Conclusiones}

La caracterización sociodemográfica de los pacientes con nódulos tiroideos, comparte características descritas en la literatura, siendo más común en las mujeres y predomina en la cuarta y quinta década de la vida, en pacientes con sobrepeso. La clasificación Bethesda más frecuente sigue siendo la benigna, los nódulos malignos fueron encontrados en un $10 \%$, un poco más elevado que en la población general.

La sensibilidad y especificidad de los hallazgos ecográficos supera el $80 \%$ y el $68 \%$ respectivamente, a excepción del tamaño mayor a $1 \mathrm{~cm}$. Las adenopatías y el diámetro AP mayor al transverso son indicadores potenciales de nódulos tiroideos malignos, el tamaño mayor a un centímetro no siempre es predictor de malignidad, sin embargo, debido al tamaño de la muestra en nuestro estudio no es posible generalizarlo como un factor determinante para la realización de biopsia por aspiración con aguja fina. Por ende, se recomienda el seguimiento de los nódulos teniendo en cuenta los criterios ecográficos de malignidad y la clasificación TIRADS para tomar decisiones con respecto a las biopsias tiroideas.

\section{Agradecimientos}

A la institución prestadora de salud por el adecuado acceso a la información de historias clínicas. A nuestros asesores por contribuir de gran manera en la realización de la presente investigación. Respecto al análisis de los datos agradecemos el apoyo obtenido por parte de los profesionales encargados de la unidad de bioestadística de la Universidad Surcolombiana quienes contribuyeron, con sus conocimientos para el adecuado desarrollo del estudio.

\section{Conflictos de interés}

Los autores declaran no tener ningún conflicto de interés al realizar el presente trabajo.

\section{Referencias}

1. Haugen BR, Alexander EK, Bible KC, Doherty GM, Mandel SJ, Nikiforov YE, et al. 2015 American thyroid association management guidelines for adult patients with thyroid nodules and differentiated thyroid cancer: The American thyroid association guidelines task force on thyroid nodules and differentiated thyroid cancer. Thyroid. 2016; 26(1): 1-133. doi: 10.1089/thy.2015.0020 
2. Román-González A, Giraldo LR, Monsalve CA, Vélez A, Restrepo JG. Nódulo tiroideo, enfoque y manejo. Revisión de la literatura. Iatreia. 2013; 26(2): 197-206.

3. Horvath E, Majlis S, Rossi R, Franco C, Niedmann JP, Castro A, et al. An ultrasonogram reporting system for thyroid nodules stratifying cancer risk for clinical management. J Clin Endocrinol Metab. 2009; 94(5): 1748-1751. doi: 10.1210/jc.2008-1724

4. Anda E, Ernaga A. Protocolo de manejo clínico del nódulo tiroideo. Medicine. 2016; 12(13): 754-757. doi: http://dx.doi.org/10.1016/j.med.2016.06.005

5. Baloch ZW, LiVolsi VA, Asa SL, Rosai J, Merino MJ, Randolph G, et al. Diagnostic terminology and morphologic criteria for cytologic diagnosis of thyroid lesions: A synopsis of the national cancer institute thyroid fine-needle aspiration state of the science conference. Diagn Cytopathol. 2008; 36(6): 425-437. doi: 10.1002/dc. 20830

6. Sánchez JF. Clasificación TI-RADS de los nódulos tiroideos en base a una escala de puntuación modificada con respecto a los criterios ecográficos de malignidad. Rev argentina radiol. 2014; 78 (3): 138-148. doi: http://dx.doi.org/10.1016/j. rard.2014.07.015

7. Singaporewalla RM, Hwee J, Lang TU, Desai V. Clinico-pathological correlation of thyroid nodule ultrasound and cytology using the TIRADS and Bethesda Classifications. World J Surg. 2017; 41(7): 1807-1811. doi: 10.1007/s00268-017-3919-5

8. Rojo JA, Begoña F, Torre EM, Ardanaz E, Guevara $\mathrm{M}$, Apiñaniz EA. Aumento de la incidencia de cáncer de tiroides en Navarra. Endocrinología, Diabetes y Nutrición. 2017; 64(6): 303-309.

9. Alvi MM, Gonorazky S, Virgili E, Soler G, Fernandez M, Cauwlaert L. Microcarcinomas papilares de tiroides no incidentales. Medicina (B Aires). 2008; 68(2): 139-143.

10. Boland GW, Lee MJ, Mueller PR, mayo-Smith W, Dawson SL, Simeone JF. Efficacy of sonographically guided biopsy of thyroid masses and cervical lymph nodes. Am J Roentgenol. 1993;161(5): 10531056. doi: 10.2214/ajr.161.5.8273609

11. Kamran SC, Marqusee E, Kim MI, Frates MC, Ritner J, Peters H, et al. Thyroid nodule size and prediction of cancer. J Clin Endocrinol Metab. 2013; 98(2): 564-570. doi: 10.1210/jc.2012-2968

12. Raparia K, Min SK, Mody DR, Anton R, Amrikachi M. Clinical outcomes for suspicious category in thyroid fine-needle aspiration biopsy patient's sex and nodule size are possible predictors of malignancy. Arch Pathol Lab Med. 2009; 133(5): 787-790. doi: 10.5858/133.5.787

13. Mendelson AA, Tamilia M, Rivera J, Hier MP,
Sherman M, Garfield N, et al. Predictors of malignancy in preoperative nondiagnostic biopsies of the thyroid. J Otolaryngol - Head Neck Surg. 2009; 38(3): 395-400.

14. Rahimi M, Farshchian N, Rezaee E, Shahebrahimi $\mathrm{K}$, Madani $\mathrm{H}$. To differentiate benign from malignant thyroid nodule comparison of sonography with FNAC findings. Pakistan J Med Sci. 2013; 29(1): 77-80. doi: 10.12669/pjms.291.2595

15. Kim MJ, Kim EK, Park SI, Kim BM, Kwak JY, Kim SJ, et al. US-guided fine-needle aspiration of thyroid nodules: Indications, techniques, results. Radiographics. 2008; 28(7): 1869-1889. doi: 10.1148/rg.287085033

16. Papini E, Guglielmi R, Bianchini A, Crescenzi A, Taccogna S, Nardi F, et al. Risk of malignancy in nonpalpable thyroid nodules: Predictive value of ultrasound and color-doppler features. J Clin Endocrinol Metab. 2002; 87(5): 1941-1946. doi: 10.1210/jcem.87.5.8504

17. Chala AI, Pava R, Franco HI, Álvarez A, et al. Criterios ecográficos diagnósticos de neoplasia maligna en el nódulo tiroideo: correlación con la punción por aspiración con aguja fina y la anatomía patológica. Rev Colomb Cir. 2013; 28: 15-23.

18. Wang N, Xu Y, Ge C, Guo R, Guo K. Association of sonographically detected calcification with thyroid carcinoma. Head Neck. 2006; 28(12): 1077-1083. doi: 10.1002/hed.20481

19. Oliveira CM, Costa RA, Patrício M, Estêvão A, Graça B, Caseiro-Alves F. Sonographic Criteria Predictive of Malignant Thyroid Nodules: Which Lesions Should be Biopsied? Acad Radiol. 2018; 25(2): 213-218. doi: 10.1016/j.acra.2017.09.006

20. Dermott MM, Gauna A, de Yampey JEW. Impacto del compromiso ganglionar en el pronóstico y la evolución del carcinoma papilar de tiroides. Rev Argent Endocrinol Metab. 2017; 54(2): 51-63. doi: 10.1016/j.raem.2016.11.006

21. Moon WJ, Baek JH, Jung SL, Kim DW, Kim EK, Kim JY, et al. Ultrasonography and the ultrasoundbased management of thyroid nodules: Consensus statement and recommendations. Korean J Radiol. 2011; 12(1): 1-14. doi: 10.3348/kjr.2011.12.1.1

22. Yoon SJ, Yoon DY, Chang SK, Seo YL, Yun EJ, Choi CS, et al. "Taller-than-wide sign" of thyroid malignancy: Comparison between ultrasound and CT. Am J Roentgenol. 2010; 194(5): W420-424. doi: 10.2214/AJR.09.3376

23. Ren J, Liu B, Zhang LL, Li HY, Zhang F, Li S, et al. A taller-than-wide shape is a good predictor of papillary thyroid carcinoma in small solid nodules. $\mathrm{J}$ Ultrasound Med. 2015; 34(1): 19-26. doi: 10.7863/ ultra.34.1.19 